\title{
Information-Theoretic Implications of Constrained Cooperation in Simple Cellular Models
}

\author{
Shlomo Shamai (Shitz)* Osvaldo Simeone ${ }^{\dagger}$, Oren Somekh ${ }^{\ddagger}$, Amichai Sanderovich*, \\ Benjamin M. Zaidel*, and H. Vincent Poor ${ }^{\ddagger}$ \\ * Department of Electrical Engineering, Technion, Haifa 32000, Israel \\ $\dagger$ CWCSPR, Department of Electrical and Computer Engineering, NJIT, Newark, NJ 07102, USA \\ $\ddagger$ Department of Electrical Engineering, Princeton University, Princeton, NJ 08544, USA
}

\begin{abstract}
Recent information theoretic results on cooperation in cellular systems are reviewed, addressing both multicell processing (cooperation among base stations) and relaying (cooperation at the user level). Two central issues are addressed, namely, first multicell processing is studied with either limitedcapacity backhaul links to a central processor or only local (and finite-capacity) cooperation among neighboring cells. The role of codebook information, decoding delay and network planning (frequency reuse) are specifically highlighted along with the impact of different transmission/ reception strategies. Next, multicell processing is considered in the presence of cooperation at the user level, focusing on both out-of-band relaying via conferencing users and in-band relaying by means of dedicated relays. Nonfading and fading uplink and downlink channels adhering to simple Wyner-type, cellular system models are targeted.
\end{abstract}

\section{INTRODUCTION}

To overcome the performance limitations of conventional cellular wireless networks in terms of throughput and coverage, a key approach has been identified in the deployment of cooperative transmission/ reception strategies. The two basic approaches to cooperation prescribe its use at either the base station (BS) or mobile station (MS) levels. As far as the BS level is concerned, multi-cell processing (MCP), sometimes referred to also as "distributed antenna system", consists of joint encoding/ decoding of the signals transmitted/ received at the BSs through the exploitation of the high-capacity backbone connecting the BSs (see [1] [2] for recent surveys on MCP). Cooperation at the MS level in the context of cellular networks has been studied under different names, such as mesh, hybrid or multi-hop cellular networks, and is based on specific forms of relaying by the MSs (see, e.g., [3]).

BS and MS cooperative technologies are enabled by the presence, respectively, of inter-BS (backbone) and inter-MS links that are not exploited by conventional cellular systems for the purpose of encoding or decoding. These links can be either wireless, orthogonal or not, thus possibly affecting the interference or bandwidth budget of the network, or wired, thus possibly requiring additional deployment efforts.

Analysis of MCP (i.e., BS cooperation) has been so far mostly based on the assumption that all the BSs in the network are connected to a central processor via links of unlimited capacity. In this case, the set of BSs effectively acts as a multiantenna transmitter (downlink) or receiver (uplink) with the caveat that the antennas are geographically distributed over a large area. Since the assumption of unlimited-capacity links to a central processor is quite unrealistic for large networks, more recently, there have been attempts to alleviate this condition by considering alternative models. In [4] a model is studied where only a subset of neighboring cells is connected to the same central unit for joint processing. In [5] [6] a topological constraint is imposed in that there exist unlimited links only between adjacent cells, and message passing techniques are implemented in order to perform joint decoding in the uplink. Finally, reference [7] (see also [8]) focuses on the uplink and assumes that the links between all the BSs and a central processor have finite capacity (finitecapacity backhaul). The reader is referred to [9][10] [11] for another framework which deals with practical aspects of finite capacity backhaul cellular systems incorporating MCP.

Information-theoretic analysis of MS cooperation in cellular networks is a more recent development. References include [12] [13] where the uplink of a two-hop mesh network is studied with amplify-and-forward (AF) cooperation (halfduplex and full-duplex, respectively) and [14] (half-duplex) [15] (full-duplex) where decode-and-forward (DF) cooperation is investigated. Analysis of compress-and-forward (CF) scheme (full-duplex) in such networks is presented in [16] (a thorough tutorial on cooperation techniques can be found in [17]).

Most of the analysis on MCP is based on different variants of a simple and analytically tractable model for cellular system proposed by Wyner [18] (henceforth, the Wyner model, see also [19]). Accordingly, the cells are arranged in either an infinite linear array or in the more familiar twodimensional hexagonal pattern, and only adjacent-cell interference is present and characterized by a single gain parameter $\alpha \in(0,1]$ (see Fig. 1). In some cases, we will also refer to a variation of the regular Wyner model, called soft-handoff model, where, assuming a linear geometry, MSs are located at the border between two successive cells and thus communicate only with the two corresponding BSs. This model has been proposed in [20] (see also [21]) and later adopted in a number of works [22] - [26]. With simplicity and analytical tractability in mind, the Wyner model provides perhaps the simplest framework a cellular system that still captures the essence of phenomena such as intercell interference and fading. 


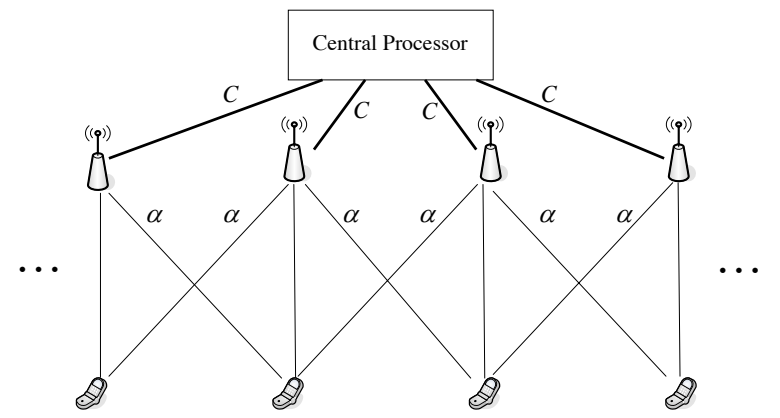

Fig. 1. Linear Wyner model with limited-capacity backhaul.

\section{THE IMPACT OF A Limited-CAPACITY BACKHAUL}

In this Section, we review recent results concerning the analysis of MCP with limited-capacity backhaul. We will focus on two cases: in the first limited-capacity backhaul links exist between each BS and a central processor (see Fig. 1), while in the second only local connectivity between adjacent BSs is allowed via finite-capacity links (see Fig. 2). In the discussion, we emphasize different assumptions regarding knowledge of codebooks (or, more generally, encoding functions) at the BSs (codebook information, CI) and treat separately uplink and downlink channels.

\section{A. Limited Backhaul to a Central Processor}

1) Uplink Channel: In [7] [8], the uplink of a Wyner model with MCP and limited-capacity backhaul, sketched in Fig. 1, was studied in two scenarios: (i) the BSs are oblivious to the codebooks used by the MSs (i.e., no CI) so that decoding is exclusively performed at the central processor; (ii) the BSs are aware of the codebooks used by the local and the nearby MSs (cluster CI).

With oblivious BSs (case $(i)$ ), the cellular uplink channel is equivalent to the setup of non-cooperative nomadic MSs communicating with a central receiver via oblivious access points with limited capacity links studied in [27][28]. Using the tools of [27] combined with the inherent symmetry of the Wyner model, an achievable per-cell sum-rate is given in [7] for Gaussian channels, and in [8] for fading channels, in the form of a simple fixed-point equation. This rate presents an SNR penalty with respect to the performance of the unlimitedcapacity setup [18], but it coincides with the cut-set bound (taken over the wireless and wired channels respectively) for high-SNR and high backhaul capacity regimes. For the low-SNR regime, [8] shows that the fixed-point equations characterizing the rate can be approximated to a closed-form solution. Using this result, the low-SNR parameters of this achievable rate, namely the minimum energy per-bit required for reliable communication and respective energy per-bit slope at zero rate [29], can be expressed as a function of the low-SNR parameters of the unlimited setup and the link capacity $C$. In addition [8] provides the rate in which the limited backhaul capacity should scale with the SNR for the achievable rate to maintain the multiplexing gain and highSNR power offset (see [30]) of the unlimited setup [18]. In the case of cluster CI (case (ii) above), an achievable rate is derived in [7][8] by allowing partial local decoding at the base stations. According to this approach, each MS splits its message and transmitted power into two parts: one is intended to be decoded locally by the in-cell BS and transmitted over the limited link to the central receiver, while the second part is processes according to the oblivious scheme and is jointly decoded by the central receiver. Other settings such as the soft-handoff model and fading channels are also considered in [8] but are not mentioned here for the sake of conciseness.

2) Downlink Channel: Turning to the downlink channel of the system in Fig. 1, MCP in the form of joint encoding of a soft-handoff Wyner-like model is studied in [32] under the assumption of finite-capacity backhaul. Similarly to [7][8], three scenarios are considered that present different tradeoffs between global processing at the central unit and local processing at the BSs and different requirements in terms of CI at the BSs: (a) local encoding with CI limited to a subset of adjacent BSs (cluster CI); (b) mixed local and central encoding with only local $\mathrm{CI}$; $(c)$ central encoding with oblivious cells (no CI). Three transmission strategies are proposed that provide achievable rates for the considered scenarios.

Let us start with the case of cluster CI (case $(a)$ ). Exploiting the local interference structure, shutting off one every $(J+2)$ th BSs forms isolated clusters of $J$ cells (see also [33]). Having the cluster's CI and messages, each BS can then perform a form of dirty paper coding (DPC) locally (under individual equal per BS power constraint [34]) and transmits its signal accordingly. In case $(b)$ of local CI, a scheme is proposed in [32] whereby each BS receives from the central unit through the limited capacity link its local user's message and a quantized version of the signal to be transmitted by its left neighboring BS. By performing local DPC, each BS is then able to cancel the interfering signal coming from its left neighboring BS. With oblivious BSs (case (c)), joint DPC under individual power constraint is performed by the central unit, which sends quantized versions of the transmitted signals to the BSs via the limited-capacity links.

The main conclusions of [32] is that central processing, even with oblivious BSs, is the preferred choice for small-tomoderate SNRs or when the backhaul capacity $C$ is allowed to increase with the SNR. On the other hand, for high SNR values and fixed capacity $C$, a system with oblivious BSs is limited by the quantization noise, and knowledge of the codebooks at the BSs becomes the factor dominating the performance. Therefore, in this regime, transmission schemes characterized by local CI or cluster CI coupled with local processing achieve better performance than central processing with oblivious cells.

\section{B. Local Connectivity}

BS cooperation in the presence of local backhaul connections between adjacent BSs is studied in [35] (see Fig. 2). 


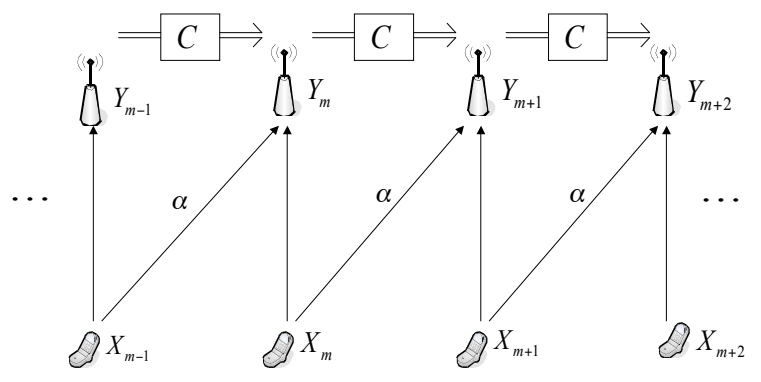

Fig. 2. Linear "Wyner-type" model with limited local backhaul connections.

Regarding CI, two scenarios are considered: (i) Local CI: Each $m$ th BS knows only the codebook used by the local ( $m$ th) MS; (ii) Two-cell CI: Each $m$ th BS knows not only the codebook used by the $m$ th MS, but also that used by the $(m-1)$ th MS. Notice that this scenario is expected to provide significantly better performance than the first, since the $(m-1)$ th MS interferes with the reception of the $m$ th $\mathrm{BS}$, and thus joint decoding techniques are known to be beneficial.

For case ( $i$ ), a first proposed technique prescribes successive decoding starting with the first $(m=0)$ BS and ending with the last $(m=M)$ BS. Successive decoding entails that, once the $m$ th BS has decoded its local message, it can compress its decided codeword via a rate- $C$ quantization codebook and send the corresponding index over the backhaul link to the $(m+1)$ th BS (Codeword Compression, CC). Reduced-delay versions of such an approach can also be devised where the maximum delay (measured in terms of the number of BSs that need to decode before a given BS is allowed to decode) is less than $M$ as for the original scheme. A second (zero-delay) scheme is also proposed that has each $m$ th BS compress and forward the received signal (instead of the decoded codeword). We define this transmission strategy as Signal Compression (SC). In the case (ii) of two-cell CI, it becomes now possible to: (a) to perform joint decoding of the local message and of (possibly part of) the interfering message at the $m$ th BS in the spirit of [36] [37]; (b) more sophisticated quantization strategies on the backhaul link that exploit the side information available at the receiving BS regarding the channel codebook [38]. This leads to a transmission strategy that is termed Decision Compression (DC) and DC with Rate Splitting (DC$\mathrm{RS})$.

As detailed in [35], the backhaul structure (local versus global as discussed in previous sections) has a major impact on the system design and performance. For instance, one of the main conclusions in references [7] [8] [32] is that BSs oblivious to the codebooks used by the MSs (as for nomadic applications) are almost optimal in the presence of global backhaul connectivity to a central processor, whereas [35] shows that major gains (even in terms of multiplexing gain)

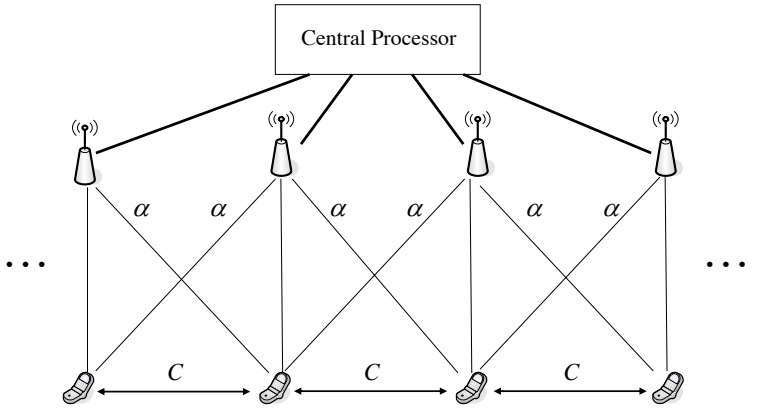

Fig. 3. Linear Wyner model with limited-capacity inter-MS conferencing channels.

can be harnessed with enhanced codebook information.

\section{THE IMPACT OF RELAYING}

We now turn to the analysis of cooperation at the MS level. We focus on two scenarios: in the first, cooperation (relaying) takes place among the MSs, while in the second relaying is afforded by the presence of dedicated relays in the system. Moreover, we distinguish between out-of-band relaying where the spectral resources used for cooperation are orthogonal to the main uplink or downlink channel and in-band relaying where the entire spectral resource is shared between uplink or downlink and relaying.

\section{A. Cooperative MSs}

1) Out-of-band Relaying: We now direct attention to a scenario where BSs perform multi-cell processing (here, with infinite-capacity backhaul), while the MSs are allowed to cooperate over finite-capacity links [44] (see Fig. 3). These links should be considered as additional spectral resources (orthogonal to the main uplink or downlink channel) that are available to enable cooperation. In modeling the interaction among MSs, the framework of conferencing encoders for the uplink [39] (see also [40][41] for related scenarios) and decoders for the downlink [38][42][43] is followed. Moreover, we focus on a scenarios with intra-cell TDMA so that conferencing channels exist only between MSs belonging to adjacent cells (inter-cell conferencing) (reference [44] also considers intra-cell conferencing for the uplink). Finally, the discussion is limited, unless stated otherwise, to Gaussian (nonfaded) channels.

Starting with the uplink and multicell decoding, an upper bound to the per-cell rate is obtained by considering a system with perfect inter-MS cooperation whereby all the MSs are able to exchange the local messages with all the other active MSs in the network. The system at hand is thus equivalent to an ISI channel with CSI at the transmitter (or equivalently an infinite MIMO system with a Toeplitz channel matrix), for which a stationary input with power spectral density obtained via standard waterfilling is known to be optimal. An achievable rate can be derived by considering an extension of the approach in [39] to multiple sources (in the spirit of [45], 


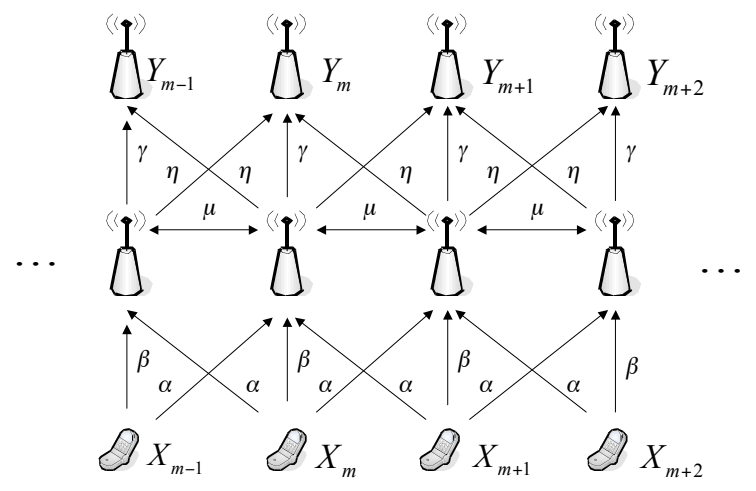

Fig. 4. Linear Wyner model with dedicated relays.

Sec. VII) [44]. Based on the observation that a stationary input is asymptotically optimal, cooperative transmission can be designed so as to implement an equivalent linear pre-filtering of the transmitted signal, which in the limit of $C \rightarrow \infty$ allows the upper bound discussed above to be attained [44].

2) In-band Relaying: Cooperation at the MS level where communications between adjacent MSs takes place by exploiting the broadcast nature of the wireless medium in the same bandwidth as the main uplink channel is studied in [49]. The model can be seen as an instance of a multiple access channel with generalized feedback, that was studied for the two-user case in [36][39][50]. Analysis and numerical results in [49] show that, even when accounting for the resources necessary to set up cooperation via inter-MS signalling, generalized feedback-based techniques can bring relevant rate gains in the low-SNR regime, and can achieve the upper bound of full cooperation if the MS measurement channels are good enough. Along these lines, the conclusions here provide a more complete picture regarding the gains from MS cooperation predicted by [44], which focused on out-of-band relaying.

\section{B. Dedicated Relays}

In this section, we briefly point to the literature on cooperation in cellular networks in the presence of dedicated relay terminals (RTs). Adhering to the simple Wyner-type setting, a mesh network with dedicated relays can be modelled as a twostage linear Wyner model with an intermediate layer of relay stations, one per cell (see Fig. 4). With coverage extension in mind we focus on users located away from the BSs. Hence, no direct link between the MSs and the BSs is assumed; the communication between the MSs and the BSs is facilitated only via the dedicated RTs.

Performance analyses of the uplink of such networks has been studied for different transmission strategies. Specifically, reference [12] considers half-duplex $\mathrm{AF}$ processing at the relays, [14] studies half-duplex DF relays, [13] full-duplex AF operation, [15] full-duplex DF, and [16] full-duplex CF. It is noted that the analyses of the full-duplex schemes consider also the deleterious effect of inter-relay interferences which can only be eliminated by $\mathrm{CF}$ relays and cooperative BSs incorporating MCP [16]. As a general conclusion, in the presence of MCP, DF techniques are only advantageous when the RTs power is sufficiently lower than the power available at the MSs. Otherwise, CF (for high RT to MS power ratio) and $\mathrm{AF}$ (for moderate RT to MS power ratio) techniques are to be preferred.

\section{CONCLUding Remarks}

Three decades after their introduction, the information theoretic understanding of cellular systems is far from being complete. In its full generality, it touches upon the most basic information theoretic models, not yet fully understood. Those comprise combinations of multiple-access, broadcast, interference and relay MIMO frequency selective fading channels, as well as fundamental network information theoretic aspects. As with the new setups at hand, which incorporates limited capacity links between BSs and among MSs, and various relaying forms, it is expected that information theory will continue to play a central role in assessing the ultimate potential and limitations of cellular networks as well as providing fundamental insights into the architecture and operation of future generations systems.

\section{ACKNOWLEDGMENT}

This research was supported by a Marie Curie Outgoing International Fellowship and the NEWCOM++ network of excellence within the 6th and 7th European Community Framework Programmes, respectively, by the U.S. National Science Foundation under Grants ANI-03-38807 and CNS-0625637, the Israel Science Foundation (ISF), and the REMON consortium for wireless communication.

\section{REFERENCES}

[1] S. Shamai (Shitz), O. Somekh, and B. M. Zaidel, "Multi-cell communications: An information theoretic perspective," in Proc. of the Joint Workshop on Commun. and Coding (JWCC'04), Donnini, Florence, Italy, Oct.14-17, 2004.

[2] O. Somekh, O. Simeone, Y. Bar-Ness, A. M. Haimovich, U. Spagnolini, and S. Shamai (Shitz), Distributed Antenna Systems: Open Architecture for Future Wireless Communications, ch. An Information Theoretic View of Distributed Antenna Processing in Cellular Systems. Auerbach Publications, CRC Press, May 2007.

[3] R. Pabst, B. H. Walke, D. C. Schultz, P. Herhold, H. Yanikomeroglu, S. Mukherjee, H. Viswanathan, M. Lott, W. Z. Sirwas, D. D. Falconer and G. P. Fettweis, "Relay-based deployment concepts for wireless and mobile broadband radio," IEEE Commun. Magazine, pp. 80-89, Sept. 2004.

[4] O. Somekh, B. M. Zaidel and S. Shamai (Shitz), "Spectral efficiency of joint multiple cell-site processors for randomly spread DS-CDMA systems," IEEE Trans. Inform. Theory, vol. 52, no. 7, pp. 2625-2637, Jul. 2007.

[5] E. Atkas, J. Evans and S. Hanly, "Distributed decoding in a cellular multiple access channel," in Proc. IEEE International Symposium on Inform. Theory (ISIT 2004), p. 484, June. 27-Jul. 2, Chicago, Illinois, 2004.

[6] O. Shental, A. J. Weiss, N. Shental and Y. Weiss, "Generalized belief propagation receiver for near optimal detection of two-dimensional channels with memory," in Proc. Inform. Theory Workshop (ITW 2004), San Antonio, Texas, Oct. 24-29, 2004. 
[7] A. Sanderovich, O. Somekh and S. Shamai (Shitz), "Uplink macro diversity with limited backhaul capacity," in Proc. IEEE International Symposium on Inform. Theory (ISIT 2007), Nice, France, Jun. 24-29, 2007.

[8] A. Sanderovich, O. Somekh and S. Shamai (Shitz), "Uplink macro diversity with limited backhaul capacity," submitted to IEEE Trans. Inform. Theory, 2008.

[9] P. Marsch, S. Khattak, and G. Fettweis, "A framework for determining realistic capacity bounds for distributed antenna systems," in Proceedings of the IEEE Inform. Theory Workshop (ITW'06), China, Oct. 2006

[10] P. Marsch and G. Fettweis, "A framework for optimizing the downlink performance of distributed antenna systems under a constrained backhaul," in Proc. European Wireless Conf. (EW 2007), Apr. 1-4, Paris, France, 2007.

[11] P. Marsch and G. Fettweis, "A Framework for Optimizing the Uplink Performance of Distributed Antenna Systems under a Constrained Backhaul," in Proc. of the IEEE International Conference on Communications (ICC 2007), Jun. 24-28, Glasgow, Scotland, 2007.

[12] O. Simeone, O. Somekh, Y. Bar-Ness and U. Spagnolini, "Uplink throughput of TDMA cellular systems with multicell processing and Amplify-and-Forward cooperation between mobiles," IEEE Trans. Wireless Commun., vol. 6, no. 8, pp. 2942-2951, Aug. 2007.

[13] O. Somekh, O. Simeone, H. V. Poor and S. Shamai, "Cellular systems with full-duplex Amplify-and-Forward relaying and cooperative base stations," in Proc. IEEE International Symp. Inform. Theory (ISIT), Nice, France, Jun. 24-29, 2007.

[14] O. Simeone, O. Somekh, Y. Bar-Ness and U. Spagnolini, "Throughput of low-power TDMA cellular systems with collaborative decoding at the base stations and cooperative transmission between mobiles", IEEE Trans. Inform. Theory, vol. 54, no. 1, pp. 459-467, Jan. 2008.

[15] O. Simeone, O. Somekh, Y. Bar-Ness, H. V. Poor and S. Shamai (Shitz), "Capacity of linear two-hop mesh networks with rate splitting, decode-and-forward relaying and cooperation," in Proc. Forty-Fifth Annual Allerton Conference on Communication, Control, and Computing, Monticello, Illinois, Sep. 26-28, 2007.

[16] O. Somekh, O. Simeone, H. V. Poor, and S. Shamai, "Cellular systems with full-duplex Compress-and-Forward relaying and cooperative base stations," in Proc. IEEE International Symp. Inform. Theory (ISIT), Toronto, Canada, Jul. 6-11, 2008

[17] G. Kramer, I. Maric and R. D. Yates, "Cooperative Communications," Foundations and Trends in Networking (FnT), Now Publishers, Jun. 2007.

[18] A. D. Wyner, "Shannon-theoretic approach to a Gaussian cellular multiple-access channel," IEEE Trans. Inform. Theory, vol. 40, pp. 1713-1727, Nov. 1994.

[19] S. V. Hanly and P. A. Whiting, "Information-theoretic capacity of multireceiver networks," Telecommun. Syst., vol. 1, pp. 1-42, 1993.

[20] O. Somekh, B. M. Zaidel, and S. Shamai (Shitz), "Sum-rate characterization of multi-cell processing," in Proc. the Canadian workshop on information theory (CWIT'05), Montreal, Canada, Jun. 5-8, 2005.

[21] O. Somekh, B. M. Zaidel, and S. Shamai (Shitz), "Sum rate characterization of joint multiple cell-site processing," IEEE Transactions on Information Theory, Vol. 53, pp. 4473-4497, Dec. 2007.

[22] Y. Liang and A. Goldsmith, "Symmetric Rate Capacity of Cellular Systems with Cooperative Base Stations," in Proc. IEEE Globecom, Nov. 27- Dec. 1, San Francisco, California, 2006.

[23] Y. Liang, T. Yoo and A. Goldsmith, "Coverage Spectral Efficiency of Cellular Systems with Cooperative Base Stations," in Proc. IEEE Globecom, Nov. 27- Dec. 1, San Francisco, California, 2006.

[24] S. Jing, D. N. C. Tse, J. Hou, J. Soriaga, J. E. Smee, and R. Padovani, "Multi-Cell Downlink Capacity with Coordinated Processing," in Proc. Information Theory and Application (ITA) Workshop, San Diego, California, Jan. 29 - Feb. 2, 2007.

[25] S. Jing, D. N. C. Tse, J. Hou, J. Soriaga, J. E. Smee, and R. Padovani, "Downlink macro-diversity in cellular networks," in Proc. IEEE International Symp. on Information Theory (ISIT'07), Nice, France, pp. 1-5, Jun. 24-29, 2007.

[26] N. Levy, O. Somekh, S. Shamai (Shitz), and O. Zeitouni, "On Certain Large Random Hermitian Jacobi Matrices with Application to Wireless Communication," submitted to IEEE Trans. Inform. Theory, 2008.

[27] A. Sanderovich, S. Shamai, Y. Steinberg, and G. Kramer, "Communication via decentralized processing," IEEE Trans. Inform. Theory, vol. 55, no. 7 , Jul. 2008
[28] A. Sanderovich, S. Shamai, Y. Steinberg, and M. Peleg, "Decentralized receiver in a MIMO system," in Proc. of IEEE International Symp. Info. Theory (ISIT'06), pp. 6-10, Seattle, WA, Jul. 2006.

[29] S. Verdú, "Spectral efficiency in the wideband regime," vol. 48, no. 6 , pp. 1329-1343, Jun. 2002.

[30] A. Lozano, A. Tulino, and S. Verdú, "High-SNR power offset in multiantenna communications," vol. 51, no. 12, pp. 4134-4151, Dec. 2005.

[31] S. Shamai and A. Wyner, "Information-theoretic considerations for symmetric cellular, multiple-access fading channels - part I,' IEEE Trans. Inform. Theory, vol. 43, no. 6, pp. 1877-1894, Nov. 1997

[32] O. Simeone, O. Somekh, S. Shamai (Shitz), and H. V. Poor, "Downlink macro-diversity with limited backhaul capacity," submitted to IEEE Trans. Wireless Com., 2008.

[33] A. Lapidoth, S. Shamai (Shitz) and M. Wigger, "A linear interference network with local side-information," in Proc. International Symposium on Inform. Theory (ISIT 2007), Nice, France, Jun. 24-29, 2007.

[34] W. Yu, "Uplink-Downlink Duality via Minimax Duality," IEEE Trans. Inform. Theory, vol. 52, no. 2, pp. 361-374, Feb. 2006.

[35] O. Simeone, O. Somekh, H. V. Poor, and S. Shamai (Shitz), “"'Enhancing uplink throughput via local base station cooperation", in Proc. Asilomar Conference on Signals, Systems and Computers, Oct. 26-29, 2008 (invited paper).

[36] A. B. Carleial, "Multiple-access channels with different generalized feedback signals," IEEE Trans. Inform. Theory, vol. 28, no. 6, pp. 841850, Nov. 1982.

[37] T. Han and K. Kobayashi, "A new achievable region for the interference channel," IEEE Trans. Inform. Theory, vol. IT-27, no. 1, pp. 49-60, Jan. 1981.

[38] S. C. Draper, B. J. Frey and F. R. Kschischang, "Interactive decoding of a broadcast message," Proc. Forty-First Annual Allerton Conference on Communication, Control, and Computing, Monticello, Illinois, 2003.

[39] F. M. J. Willems, Informationtheoretical Results for the Discrete Memoryless Multiple Access Channel, Ph.D. thesis, Katholieke Universiteit Leuven, 1982.

[40] I. Maric, R. D. Yates and G. Kramer, "The discrete memoryless compound multiple access channel with conferencing encoders," in Proc. International Symposium on Inform. Theory (ISIT 2005), pp. 407-410, Adelaide, Australia, Sep. 4-9, 2005.

[41] C. T. K. Ng, I. Maric, A. J. Goldsmith, S. Shamai (Shitz), and R. D. Yates, "Iterative and one-shot conferencing in relay channels," in Proc. IEEE Information Theory Workshop (ITW 2006), Punta del Este, Uruguay, Mar. 13-17.

[42] R. Dabora and S. Servetto, "Broadcast channels with cooperating decoders," IEEE Trans. Inform. Theory, vol. 52, no. 12, pp. 5438-5454, Dec. 2006.

[43] Y. Liang and V. V. Veeravalli, "Cooperative relay broadcast channels," IEEE Trans. Inform. Theory, vol. 53, no. 3, pp. 900-928, Mar. 2007.

[44] O. Simeone, O. Somekh, G. Kramer, H. V. Poor, and S. Shamai (Shitz), "Throughput of cellular systems with conferencing mobiles and cooperative base-stations," Eurasip Journal on Wireless Commun. and Networking, May. 2008.

[45] D. Slepian and J. K. Wolf, "A coding theorem for multiple access channels with correlated sources," Bell Systems Tech. J., vol. 52, pp. 1037-1076, Sep. 1973.

[46] T. Guess and M. K. Varanasi, "An information-theoretic framework for deriving canonical decision-feedback receivers in Gaussian channels," IEEE Trans. Inform. Theory, vol. 51, no. 1, pp. 173-187, Jan. 2005.

[47] R. Zamir, S. Shamai and U. Erez, "Nested linear/ lattice codes for structured multiterminal binning," IEEE Trans. Inform. Theory, vol. 48 , no. 6, pp. 1250-1276, Jun. 2002.

[48] Wei Yu and J. M. Cioffi, "Sum capacity of Gaussian vector broadcast channels," IEEE Trans. Inform. Theory, vol. 50, no. 9, pp. 1875-1892, Sep. 2004.

[49] O. Simeone, O. Somekh, G. Kramer, H. V. Poor and S. Shamai (Shitz), "Uplink sum-rate analysis of a multi-cell system with feedback," submitted to the Allerton Conference on Communication, Control, and Computing, 2008.

[50] R. C. King, Multiple access channels with generalized feedback, $\mathrm{PhD}$ thesis, Stanford Univ., CA, Mar. 1978.

[51] O. Simeone, O. Somekh, H. V. Poor, and S. Shamai (Shitz), "Distributed MIMO systems with oblivious antennas," in Proc. IEEE International Symposium Inform. Theory (ISIT 2008), Toronto, Canada, Jul. 6-11, 2008. 